\title{
Biometría del "churrete austral", Cinclodes antarcticus (Garnot) en isla Gonzalo, archipiélago Diego Ramírez, sur de Chile
}

\section{Biometry of "Blackish Cinclodes", Cinclodes antarcticus (Garnot) at Gonzalo island, Diego Ramirez archipelago, southern Chile}

\author{
Jaime A. Cursach ${ }^{1,2^{*}}$, Cristián G. Suazo ${ }^{3}$, Claudio N. Tobar ${ }^{1}$ \& Jaime R. Rau ${ }^{1,4}$ \\ ${ }^{1}$ Laboratorio de Ecología, Departamento de Ciencias Básicas, Universidad de Los Lagos, casilla 933, Osorno, Chile. \\ ${ }^{2}$ ATLAS. Programa de Investigación Interdisciplinaria en Complejidad Territorial y Sustentabilidad, Departamento de Ciencias \\ Sociales, Universidad de Los Lagos, casilla 933, Osorno, Chile. \\ ${ }^{3}$ Albatross Task Force - Chile, BirdLife International. \\ ${ }^{4}$ Programa IBAM, Universidad de Los Lagos, casilla 933, Osorno, Chile. \\ *E-mail: jcurval@gmail.com
}

\begin{abstract}
RESUMEN
Durante enero 2011 se registraron datos morfométricos y conductuales de Cinclodes antarcticus en isla Gonzalo, Diego Ramírez, sur de Chile. La longitud del pico fue significativamente mayor en machos respecto de hembras. C. antarcticus es un ave comensalista de colonias reproductivas de aves y mamíferos marinos de la Patagonia austral.
\end{abstract}

\begin{abstract}
During january 2011 we recorded morphometric and behavioral data of Cinclodes antarcticus in Gonzalo island, Diego Ramirez, southern Chile. The bill length was significantly higher in males compared to females. C. antarcticus is a commensal bird of seabird and marine mammal colonies of the Patagonia.
\end{abstract}

El churrete austral Cinclodes antarcticus (Garnot, 1826) es un ave endémica del sur de Sudamérica, considerado uno de los Paseriformes más marítimos que se conocen, habitando islas australes expuestas al océano, asociándose su presencia a loberías y colonias de aves marinas (Venegas \& Sielfeld 1979, Araya \& Millie 2005, BirdLife International 2011). La información publicada sobre el churrete austral es escasa, la gran mayoría de ella se refiere a cortas descripciones de su plumaje en guías de identificación (e.g. Couve \& Vidal 2003, Araya \& Millie 2005) y de breves referencias sobre su distribución, filogenia, alimentación y zoonosis (Venegas \& Sielfeld 1979, Robbins et al. 2001, Chesser 2004, Kusch et al. 2007). El presente trabajo aporta los primeros datos publicados sobre la biometría del churrete austral en territorio chileno, analizando diferencias entre sexos y algunas observaciones sobre su comportamiento y alimentación.

El trabajo se realizó en isla Gonzalo $\left(56^{\circ} 31^{\prime} 16,8^{\prime}\right.$ 'S; $68^{\circ} 42^{\prime} 53,5^{\prime \prime} \mathrm{O}$ ), ubicadaen elmargenaustral delarchipiélago Diego Ramírez, sur de Chile. Dicho archipiélago se ubica a $112 \mathrm{~km}$ al suroeste del Cabo de Hornos y representa geográficamente el extremo más austral de América del Sur (Schlatter \& Riveros 1997). Entre los días 3 y 27 de enero 2011 se capturaron 31 churretes australes mediante red de niebla instalada entre la vegetación de coirón Poa flabellata [(Lam.) Hook f.]. Todos los ejemplares se marcaron individualmente mediante corte apical de la décima primaria del ala derecha de cada ave, evitando así el doble muestreo. De cada ejemplar se tomaron las siguientes medidas: peso $( \pm 40 \mathrm{~g})$, longitud de tarso $( \pm 0,1$ $\mathrm{mm})$, longitud de pico $( \pm 0,1 \mathrm{~mm})$, longitud total $( \pm 0,5$ $\mathrm{mm})$, longitud de la cola $( \pm 0,5 \mathrm{~mm})$, longitud de cuerda máxima alar $( \pm 0,5 \mathrm{~mm})$, longitud de la novena primaria $( \pm$ $0,5 \mathrm{~mm})$ y longitud de la cuarta primaria $( \pm 0,5 \mathrm{~mm})$, estas últimas tres mediciones fueron realizadas en el ala derecha de cada individuo. Se registró el sexo y estado de madurez sexual de cada ave en función a la condición cloacal y/o placa incubatriz según fuese el caso, clasificando su estado sexual como inmaduro, activo y post-reproductivo (North American Banding Council 2003). De forma paralela se realizaron observaciones directas sobre la conducta y alimentación del churrete austral en isla Gonzalo. Para determinar diferencias estadísticas en las mediciones 
corporales entre hembras y machos de esta especie se utilizó la prueba $\mathrm{t}$ de Student para datos independientes, tras comprobar el ajuste a la distribución normal mediante prueba de Kolmogorov-Smirnov $(\mathrm{P}<0,05)$.

En total se capturaron 31 ejemplares, de los cuales 10 $(32,3 \%)$ fueron hembras; incluyendo a tres ejemplares con señales de inmadurez sexual, seis activas y una en estado post-reproductivo (Tabla 1). Los individuos machos capturados fueron 21 (67,7\%), de los cuales seis se encontraban sexualmente inmaduros, 11 activos y cuatro en estado post-reproductivo (Tabla 2).

De todos los registros morfométricos en individuos sexualmente maduros realizados entre ambos sexos, sólo la longitud del pico presentó diferencias significativas $(\mathrm{t}=$ 2,14; g.l $=14 ; \mathrm{P}<0,05)$, siendo mayor en el macho.
La actividad del churrete austral en isla Gonzalo fue principalmente diurna, mostrándose muy confiado a la presencia humana, conducta ya reportada por Cawkell \& Hamilton (1961) en islas Malvinas (51 ${ }^{\circ} 43^{\prime} \mathrm{S}$; $61^{\circ} 18^{\prime} \mathrm{O}$ ), lugar en donde se conoce a esta ave por tomar la comida de los seres humanos. De forma similar a lo registrado por Robbins et al. (2001), el churrete austral se alimenta de invertebrados (i.e. anfípodos marinos y moluscos) presentes entre las algas (e.g. género Macrocystis) varadas en la playa de isla Gonzalo, así como también de invertebrados que captura entre el denso follaje de coirón. De manera oportunista se les observó capturando ectoparásitos (i.e. garrapatas del género Ixodes) presentes en nidos de albatros de ceja negra Thalassarche melanophrys (Temminck, 1839) y albatros de cabeza gris $T$. chrysostoma (Foster, 1785), junto con alimentarse de los regurgitados de ambos albatros, constituidos principalmente por restos digeridos de peces, cefalópodos y krill Euphausia superba (Dana, 1850). Lo

TABla 1. Promedio $( \pm \mathrm{DE})$ de las diferentes mediciones corporales en hembras $(n=10)$ de $C$. antarcticus con diferentes estados de madurez sexual, en isla Gonzalo, Diego Ramírez, Chile.

TABLE 1. Average $( \pm \mathrm{SD})$ of different body measurements in females $(\mathrm{n}=10)$ of $C$. antarcticus at different stages of sexual maturity, in Gonzalo island, Diego Ramirez, Chile.

\begin{tabular}{|c|c|c|c|}
\hline Caracteres & $\begin{array}{c}\text { inmaduras }(n=3) \\
\text { Media } \pm \mathbf{D E}\end{array}$ & $\begin{array}{c}\text { activas }(n=6) \\
\text { Media } \pm \mathbf{D E}\end{array}$ & $\begin{array}{c}\text { post-reproductiva }(\mathrm{n}=1) \\
\mathbf{M e d i a} \pm \mathbf{D E}\end{array}$ \\
\hline Peso (g) & $63,2 \pm 2,1$ & $66,5 \pm 6,0$ & $56,7 \pm 0,0$ \\
\hline Longitud de tarso (mm) & $29,3 \pm 0,8$ & $29,9 \pm 0,5$ & $29,5 \pm 0,0$ \\
\hline longitud de pico (mm) & $16,3 \pm 0,5$ & $17,1 \pm 1,1$ & $16,9 \pm 0,0$ \\
\hline longitud total (cm) & $20,8 \pm 0,5$ & $21,4 \pm 0,7$ & $20,5 \pm 0,0$ \\
\hline longitud de la cola $(\mathrm{cm})$ & $8,0 \pm 0,3$ & $8,3 \pm 0,5$ & $9,2 \pm 0,0$ \\
\hline longitud de ala $(\mathrm{cm})$ & $10,9 \pm 0,4$ & $11,1 \pm 0,3$ & $10,6 \pm 0,0$ \\
\hline longitud de novena primaria $(\mathrm{cm})$ & $8,3 \pm 0,6$ & $7,9 \pm 0,3$ & $8,1 \pm 0,0$ \\
\hline longitud de cuarta primaria $(\mathrm{cm})$ & $7,5 \pm 0,3$ & $7,5 \pm 0,2$ & $7,4 \pm 0,0$ \\
\hline
\end{tabular}

Tabla 2. Promedio $( \pm \mathrm{DE})$ de las diferentes mediciones corporales en machos $(\mathrm{n}=21)$ de $C$. antarcticus con diferentes estados de madurez sexual, en isla Gonzalo, Diego Ramírez, Chile.

TABLE 2. Average $( \pm \mathrm{SD})$ of different body measurements in males $(n=21)$ of $C$. antarcticus at different stages of sexual maturity, in Gonzalo island, Diego Ramirez, Chile.

\begin{tabular}{|c|c|c|c|}
\hline Caracteres & $\begin{array}{c}\text { inmaduros }(\mathrm{n}=6) \\
\text { Media } \pm \mathbf{D E}\end{array}$ & $\begin{array}{c}\text { activos }(n=11) \\
\text { Media } \pm \text { DE }\end{array}$ & $\begin{array}{c}\text { post-reproductivo }(\mathrm{n}=4) \\
\mathbf{M e d i a} \pm \mathbf{D E}\end{array}$ \\
\hline Peso (g) & $62,0 \pm 1,6$ & $60,1 \pm 3,1$ & $61,9 \pm 2,1$ \\
\hline Longitud del tarso (mm) & $30,1 \pm 0,6$ & $30,1 \pm 0,9$ & $30,3 \pm 0,7$ \\
\hline longitud del pico (mm) & $16,7 \pm 0,8$ & $19,1 \pm 1,7$ & $17,3 \pm 0,3$ \\
\hline longitud total $(\mathrm{cm})$ & $20,7 \pm 0,6$ & $21,1 \pm 0,9$ & $20,9 \pm 0,4$ \\
\hline longitud de la cola $(\mathrm{cm})$ & $8,4 \pm 0,5$ & $8,7 \pm 0,6$ & $8,8 \pm 0,6$ \\
\hline longitud del ala $(\mathrm{cm})$ & $11,0 \pm 0,2$ & $12,1 \pm 2,7$ & $10,9 \pm 0,5$ \\
\hline longitud de la novena primaria $(\mathrm{cm})$ & $8,1 \pm 0,2$ & $8,2 \pm 0,4$ & $7,9 \pm 0,3$ \\
\hline longitud de la cuarta primaria $(\mathrm{cm})$ & $7,3 \pm 0,1$ & $7,3 \pm 1,1$ & $7,0 \pm 0,2$ \\
\hline
\end{tabular}


anterior, confirma al churrete austral en la categoría de ave comensalista de colonias reproductivas de aves y mamíferos marinos de la Patagonia austral (Robbins et al. 2001).

En isla Gonzalo el churrete austral nidifica en cuevas que les permiten mantenerse como residentes en la isla durante todo el año, refugiándose del agresivo clima invernal (Schlatter \& Riveros 1997). Junto a las islas Ildefonso (5548' S; 69²4' O) (Clark 2008) e isla Noir (54²8' S; $73^{\circ} 00^{\prime} \mathrm{O}$ ) (Kusch et al. 2007), isla Gonzalo es el tercer sitio en donde se ha reportado la residencia del churrete austral en la Patagonia chilena.

Los presentes registros morfométricos del churrete austral indicaron que su peso corporal se mantuvo en un rango similar al registrado por Schlatter \& Riveros (1997) en isla Gonzalo durante el año 1981. Al comparar las mediciones corporales del churrete austral con los datos publicados para otras especies de Cinclodes que habitan en Chile, se observó que el peso corporal del churrete austral es mayor al de Cinclodes patagonicus (Gmelin, 1789), C. oustaleti (Scott, 1900) y C. fuscus (Vieillot, 1818), pero a la vez menor al de individuos de C. nigrofumosus (d' Orbigny) de la zona central de Chile (30-38 S) (Morgado et al. 1987, Sabat et al. 2006). Además, las morfometrías reportadas para $C$. taczanowskii (Berlepsch \& Stolzmann, 1892) en la costa sur de Perú $\left(15^{\circ} \mathrm{S}\right)$ indican que su peso corporal, longitud de ala y de tarso son similares a las del churrete austral (Bertolero \& Zavalaga 2003). Aún así, la escasez de datos publicados sobre la biometría del churrete austral así como también de otras representantes del género Cinclodes que habitan ambientes costeros impide realizar comparaciones ecomorfológicas a diferentes latitudes.

\section{AGRADECIMIENTOS}

A la Dirección de Investigación de la Universidad de Los Lagos. A la Armada de Chile. J.A. Cursach agradece el apoyo de la beca Nibaldo Bahamonde (U Lagos). C.G. Suazo agradece el apoyo de CONICYT-Chile y Pacific Seabird Group.

\section{BIBLIOGRAFÍA}

Araya, B. \& Millie, G. 2005. Guía de campo de las aves de Chile. Editorial Universitaria, Santiago. 406 pp.

Bertolero, A. \& Zavalaga, C. 2003. Observaciones sobre la biometría y la muda del churrete marisquero (Cinclodes taczanowskii) en Punta San Juan, costa sur de Perú. Ornitología Neotropical 14:469-475.

BirdLife International. 2011. Species factsheet: Cinclodes antarcticus. URL: http://www.birdlife.org. Accesado: Junio 17, 2011.

Cawkell, E.M. \& Hamilton, J.E. 1961. The birds of the Falkland Islands. Ibis 103:1-27.

Chesser, R.T. 2004. Systematics, evolution, and biogeography of the South American ovenbird genus Cinclodes. The Auk 121:752-766.

Clark, G. 2008. La travesía del Totorore. Editorial El MercurioAguilar S.A., Santiago. 622 pp.

Couve, E. \& Vidal, C. 2003. Birds of Patagonia, Tierra del Fuego \& Antartic Peninsula. Editorial Fantástico Sur Birding Ltda., Punta Arenas. 656 pp.

Kusch, A., Marín, M., Oheler, D. \& Drieschman, S. 2007. Notas sobre la avifauna de isla Noir $\left(54^{\circ} 28^{\prime} \mathrm{S}-73^{\circ} 00^{\prime} \mathrm{W}\right)$. Anales del Instituto de la Patagonia 35:61-66.

Morgado, E., Günther, B. \& Gonzalez, U. 1987. On the allometry of Kings. Revista Chilena de Historia Natural 60:71-79.

North American Banding Council. 2003. Manual para anillar Paseriformes y cuasi Paseriformes del anillador de Norteamérica (excluyendo colibríes y búhos). URL: http:// www.nabanding.net/manuals/PasserSpa.pdf. Accesado: Enero 02, 2011.

Robbins, R.G., Sorkin, L.N. \& Vuilleumier, F. 2001. First report of Ixodes auritulus Neumann (Acari: Ixodida: Ixodidae) from the blackish cinclodes, Cinclodes antarcticus (Garnot) (Aves: Passeriformes: Furnariidae), with additional records of parasitism of Cinclodes spp. by this tick species. Proceedings of the Entomological Society of Washington 103:265-268.

Sabat, P., Maldonado, K., Fariña, J.M. \& Martínez del Río, C. 2006. Osmoregulatory capacity and the ability to use marine food sources in two coastal songbirds (Cinclodes: Furnariidae) along a latitudinal gradient. Oecología 148:250-257.

Schlatter, R.P. \& Riveros, G. 1997. Historia natural del Archipiélago Diego Ramírez, Chile. Serie Científica INACH 47:87-112.

Venegas, C. \& Sielfeld, W. 1979. Antecedentes para la determinación de un nuevo distrito zoogeográfico en el litoral exterior de Magallanes. Anales del Instituto de la Patagonia 10:201-208.

Recibido: 08.08.11

Aceptado: 29.02.12 